\title{
DUAL FLOW BIOREACTOR WITH ULTRATHIN MICROPOROUS TEER SENSING MEMBRANE FOR EVALUATION OF NANOPARTICLE TOXICITY
}

\author{
Tommaso Sbrana $^{1 \dagger}$, Nadia Ucciferri ${ }^{1,2 \dagger}$, Mèlanie Favrè ${ }^{3 \dagger}$, Sher Ahmed ${ }^{3}$, Eva-Maria \\ Collnot $^{2}$, Claus-Michael Lehr ${ }^{2}$, Arti Ahluwalia ${ }^{1}$, Martha Liley ${ }^{3}$ \\ 1 Research Center "E. Piaggio", via Diotisalvi, 2, University of Pisa, Pisa 56126, Italy. \\ 2 Helmholtz-Institute for Pharmaceutical Research Saarland (HIPS), Saarland University, A \\ 4 1, 66123 Saarbrücken, Germany. \\ 3 Swiss Center for Electronics and Microtechnology, Jaquet Droz 1, CH 2002 Neuchâtel, \\ Switzerland. \\ $\dagger$ These authors contributed equally to this work. \\ * Author to whom correspondence should be addressed; E-Mail: arti.ahluwalia@ unipi.it, \\ Phone: 0039050 2217050, Fax 00390502217051
}

\begin{abstract}
:
Permeability studies across biological barriers are of primary importance in drug delivery as well as in toxicology when investigating the absorption and translocation of a substance. The study of nanomaterial interaction with epithelial barriers is of particular interest given their growing use in nanomedicine as well as concerns about their potential hazard. Here we describe the design and fabrication of a new bioreactor with an ultrathin microporous sensing support for the study of nanoparticle toxicity in intestinal epithelial cells in conditions which better recapitulate the physiological environment. Thanks to the integration of 4 electrodes in the microporous membrane, the system allows real-time and continuous sensing of TEER (trans epithelial electrical resistance) during flow without interruption or perturbation of experiments. The TEER bioreactor was tested using Caco-2 cells as an in vitro model of intestinal epithelia. When exposed to silver nanoparticles, which are known to be toxic, the embedded electrodes enabled non-invasive evaluation of barrier impairment over time. This device can be used to study barrier integrity and the kinetics of nanomaterial induced damage to epithelial barriers in physiologically relevant conditions.
\end{abstract}

Keywords: microfabrication; TEER sensor; nanomaterials; fluidics; bioreactor. 


\section{Introduction}

The capacity of biological membranes to restrict the passage of exogenous molecules is the basis of the defence mechanism of the human body which tries to keep out foreign materials. This feature, in fact, limits the movement of solutes and pathogens in order to prevent them reaching the systemic circulation. On the other hand, overcoming these barriers is one of the major challenges in the field of drug delivery, where the aim is actually to enhance the transport of drugs in order to have therapeutic effects while minimizing the applied dose [1]. The study of nanoparticle (NP) translocation across biological barriers is of interest to both toxicology and drug delivery research, as these new materials are promising carriers of biologicals or pharmaceuticals [2]. Unfortunately, the very same properties that enable their passage across biological barriers such as lung, intestine and skin and into downstream cells and organs, can also lead to toxic effects. Currently there is major concern about the hazards of nanomaterials and a number of regulatory bodies and public groups have called for the development of appropriate physiologically-relevant in vitro methods for the assessment of NP hazard [3].

An important route of entry for nanomaterials is the gut, particularly in the case of ingestible NPs such as silver. The intestine is a dynamic organ; the flow of digested food and intestinal fluids on the luminal side contributes to the absorption and passage of substances across the epithelial barrier, while blood flow on the adventitial side is responsible for their subsequent distribution. Indeed, it is well-known that flow enhances cell metabolic function and cytoskeletal organization in most cell types [4], and that barrier permeability changes under physiological-like shear stress [5]. Although it is widely accepted that static conditions do not mimic the physiological environment of biological barriers, the most common in vitro model for the study of intestinal permeation, NP passage or drug delivery is a monolayer of differentiated Caco-2 cells on polymeric (polycarbonate, polystyrene) transwell inserts, in the absence of any convective movement [6].

There is also some concern about the suitability of commercially-available polymer membranes for the study of nanomaterial transport across barrier models because of their tendency to adhere to hydrophobic materials and to clog porous inserts [7]. In addition, commercially available inserts have relatively thick porous membranes with high pore tortuosity leading to long times for particles to cross the membrane, which can give a misleadingly low permeability profile.

The integrity of model biological barriers is often evaluated through TEER (trans epithelial electrical resistance) measurements. The electrical resistance of a confluent layer of epithelial cells is used as a measure of the tightness of the intercellular junctions. Amongst the main advantages of the TEER approach are its ease and non-destructive nature [8]. Different

methods may be used to measure TEER. The most widely employed system is the EVOM ${ }^{2}$ (World Precision Instrument, Sarasota SL USA), which uses a "chopstick" electrode configuration to measure TEER at discrete, widely-spaced time intervals. Electrodes are carefully placed in transwell and 4-point measurement of electrical resistance is made with an 
alternating current $(10 \mu \mathrm{A}, 12.5 \mathrm{~Hz})$. Here, one pair of electrodes injects a known current, while the second pair of electrodes measures the voltage across the cell layer. This system effectively eliminates contact effects as well as the capacitive contribution of the cell monolayer. There have been a few reports of TEER in fluidic systems. Chopsticks and otherwise exposed electrodes can be very sensitive to flow, as the movement of ions around them gives rise to measurement artefacts. So some investigators have performed measurements using a stopped flow approach [9]. Microfluidic systems with permanently embedded electrodes may also reduce the instability problem; however the data reported by Ferrell et al. [10] still have rather large errorbars and TEER is acquired by attaching the electrodes to an EVOM at 24 hour intervals. An interesting and unique system realized by Cucullo et al. [11] uses a four electrode sensor integrated in a mini hollow fibre system, in which endothelial cells line the lumen of the fibres, through which media flows. Known as Flocel (Flocel Inc. Cleveland, OH, USA), the system uses impedance measurements (0.06 V swept over a range of frequencies) and models are used to extract the TEER and cell layer capacitance in real time. Unfortunately the hollow fibre configuration is unwieldy and does not allow for cell imaging.

Here we describe a new fluidic device with an integrated microfabricated TEER sensing membrane which permits real time and continuous monitoring of epithelial monolayers under physiologically-relevant flow conditions. Given the unsuitability of commercial transwelltype membranes for the study of nanoparticle passage across epithelial barriers, we replaced the polymeric porous membrane with a microfabricated silicon nitride microporous membrane in a silicon chip. As TEER electrodes may be sensitive to flow, contacts were directly integrated into the microfabricated membrane. The bioreactor chamber was designed so that the membrane divided it into two compartments, each of which is connected to a separate fluidic channel. The aim of this work is to report the design and validation of the device and its application to evaluation of NP toxicity and barrier impairment in an in vitro model of the intestinal epithelium using the Caco-2 cell line.

\section{Design and Engineering of the TEER Bioreactor}

\subsection{Micro fabricated ultrathin porous support}

The porous cell culture supports are fabricated using standard microfabrication processes. Briefly, $500 \mathrm{~nm}$ of low stress silicon nitride is deposited on both sides of a $380 \mu \mathrm{m}$ thick silicon wafer. Photolithography and dry etching define the pore size, shape and density on the front side of the wafer and square openings on the back side (Figure 1a). The square openings serve as a mask for the $\mathrm{KOH}$ etch used to release the square porous membranes $1 \mathrm{x} 1 \mathrm{~mm}$ in size. The wafer is then diced into $14 \times 14 \mathrm{~mm}$ chips each of which has 23 square porous membranes with a fill factor of $15 \%$ (Figure 1b). The total membrane surface area is thus 23 
$\mathrm{mm}^{2}$. The pores are $1 \mu \mathrm{m}$ in diameter and have a tortuosity ${ }^{1}$ of 1 providing direct access from the apical to basal side (Figure 1c).

In order to perform electrical measurements, two platinum electrodes are deposited on the silicon wafer. There are two contacts on each side of the chip, to allow for a 4 probe TEER measurement (Figure 1b).

Previous work by the authors has demonstrated that these ultrathin porous supports are suitable for epithelial cell culture. Moreover, in the absence of cells, NPs translocate through the membrane with significantly less clogging than in transwell inserts, which typically have high pore tortuosity [12].

\subsection{The fluidic chambers}

The fluidic chamber is based on the single compartment bioreactor reported in Mazzei et al. [13] and its two compartment, two-flow module for epithelial cultures described in Giusti et al. [14]. In the latter bioreactor Caco-2 intestinal epithelial cells were shown to possess increased TEER values and enhanced fluorescein passage upon exposure to apical and basal medium flow. Two modifications have been made to adapt the system to integrated TEER sensing: the housing of a polycarbonate holder plus membrane; and holes for the insertion of 4 gold contact rods. The polycarbonate holder (described in the Supplementary Materials) was fabricated for handling of the porous support during cell culture and for facile insertion into the bioreactor.

As shown in Figure 2a, the chamber consists of two compartments (respectively the apical and the basal) fabricated from PDMS. Further details on the chamber design and fabrication are described in the Supplementary Materials. Once fully assembled the TEER bioreactor is 3 $\mathrm{cm}$ in diameter and $4 \mathrm{~cm}$ in height and can be easily moved and handled for connection to an EVOM (Figure 2b). Each half chamber is connected to a pump (IPC-4, Ismatech, CH) and a mixing bottle, which serves as a priming volume and as a reservoir for oxygenation and medium sampling. Each circuit contains $15 \mathrm{~mL}$ of liquid.

\subsection{TransEpithelial Electrical Resistance (TEER) Sensing}

Four spring-loaded gold probes $(19 \mathrm{~mm}$ long, $0.25 \mathrm{~mm}$ diameter, Everett Charles Technologies, Pomona CA, USA) were used to contact the platinum electrodes. The probes were connected by wires to an EVOM2 and used to measure TEER using a 4 point configuration, according to the equivalent circuit represented in Figure 2c. As explained in

\footnotetext{
${ }^{1}$ Defined here as the so-called hydraulic tortusoity or elongation of path length with respect to a straight line, such that a tortuosity of 1 is a direct linear path. All other paths have a tortuosity $>1[18]$.
} 
the Supplementary Materials the 4 probes were connected to the EVOM. The BNC output of the EVOM was connected to a computer via an acquisition card (NI USB-6008, National Instrument) to enable continuous TEER sensing (1 reading per minute). The data were acquired using dedicated software developed using LabVIEW (National Instrument, Austin, USA). Briefly every minute, $1 \mathrm{~s}$ of data were acquired at a frequency of $200 \mathrm{~Hz}$, filtered and averaged.

\section{$2.4 \quad$ Modelling}

Flow conditions in the TEER bioreactor were determined using Finite Element Methods (FEM). In particular we analysed flow-mediated transport using COMSOL Multiphysics (COSMOL AB, Stockholm, Sweden, version 3.4), using the same methods described in Giusti et al. [14]. The wall shear stress in the human intestine is $2.10^{-4}-8.10^{-3} \mathrm{~Pa}$ [15]. Therefore the apical flow rate was selected to so as mimic this stress on the surface of the membrane. Having established the apical flow rate, the basal flow was selected to maximize the density of velocity streamlines through the membrane and into the lower circuit without vortices. Using these criteria, the flow rates were $200 \mu \mathrm{L} / \mathrm{min}$ in the top circuit (giving a maximum shear stress of $1.68 .10^{-3} \mathrm{~Pa}$ on the membrane) and $100 \mu \mathrm{L} / \mathrm{min}$ in the bottom circuit (Figure 3a and $\mathrm{b}$ ). These flow rates were used for all the experiments reported.

\section{Materials and Methods}

\subsection{TEER sensor validation}

The TEER of an epithelial barrier is expressed in $\Omega . \mathrm{cm}^{2}$, and is the measured resistance in the presence of cells subtracted from the resistance of a blank support, multiplied by the area of the membrane. To test the accuracy of TEER sensing in the presence of flow, we first performed a series of direct resistance measurements using salt solution. The polycarbonate holder containing the cell culture support chip without cells was placed in the bioreactor. Fifteen $\mathrm{mL}$ of $\mathrm{NaCl}$ in deionized water were added to each mixing bottle; the system was clamped and electrodes were inserted till electrical contact was made with the chip. The circuits were filled by pumping the solution from the mixing bottle using the flow rates established from the FEM models. Preliminary tests were performed using a $0.8 \% \mathrm{w} / \mathrm{v}$ solution (the concentration in physiological saline), measuring resistance without flow for 60 min and then measuring it for further 60 min with flow, simply switching on the pump.

Then, to calibrate the system, the resistance was evaluated for different concentrations of $\mathrm{NaCl}$ (from 0.05 to $10 \% \mathrm{w} / \mathrm{v}$ ) in both static conditions (pump off) and in dynamic conditions (pump on). The values obtained in the bioreactor were compared with values measured in equivalent salt solutions using EVOM "chopsticks" in Transwell inserts. 


\subsection{Caco-2 cultures}

The Caco-2 cell line is derived from human epithelial colorectal adenocarcinoma and is a widely accepted in vitro model of the human small intestine. After 21 days of culture on porous substrates the cells become differentiate to an enterocyte-like phenotype, expressing tight junctions and microvilli [6]. Caco-2 cells from clone C2Bbe1 (ATCC Culture, USA) were cultured in Dulbecco's modified Eagle's (DMEM) High-Glucose medium, 10\% Foetal bovine serum (FBS), 1\% Penicillin/Streptomycin, 1\% non-essential amino acids (all from PAA, Pashing, Austria). The microfabricated porous supports were sterilized in their holders by autoclaving and placed in 6 well plates before seeding with 60,000 cells $/ \mathrm{cm}^{2}$. The cells adhered and began to spread on the supports after a few hours. After 21 days in a cell culture incubator $\left(37^{\circ} \mathrm{C}, 5 \% \quad \mathrm{CO}_{2}\right)$, when the cells had attained confluence and formed a differentiated epithelial barrier as ascertained from microscopic observation and TEER value, the holder was ready for insertion into the TEER bioreactor.

\subsection{Nanoparticles}

Two different NPs known to possess, respectively, low and high toxicity in standard static cell culture were used to characterize cell response and evaluate the corresponding TEER data. Polystyrene-FITC (Fluorescein isothiocyanate) Fluoresbrite ${ }^{\circledR}$ (PS) nanoparticles ( Polyscience Inc., Germany, nominal diameter $55 \mathrm{~nm}$ ) were used at a concentration of 1 $\mathrm{mg} / \mathrm{mL}$ in complete DMEM. At this concentration the particles are nontoxic. Silver nanoparticles (Ag, NM300 from Ras $\mathrm{GmbH}$, an OECD referenced nanomaterial with a nominal diameter of $20 \mathrm{~nm}$ ), were used at a concentration of $50 \mu \mathrm{g} / \mathrm{mL}$, which is approximately the reported LC50 value [16]. Details of NP characterization are provided in the Supplementary Materials.

\subsection{Cell culture and NP experiments}

For the cell culture experiments NPs were added to the mixing bottle in the upper circuit. The system was closed and filled as described for the salt solution experiments, and let run for 24 $\mathrm{h}$ in the incubator; during this time TEER values were acquired and registered as described above.

Control experiments were performed with the same dynamic set up but in absence of NPs. In order to evaluate the ability of the system to sense or detect a drop in barrier electrical resistance, Triton-X was used as a positive control for barrier disruption. 0.1\% Triton-X100 (Sigma-Aldrich, St. Louis, USA) was added in the upper circuit bottle. In addition, a static control was also performed with cells on the microfabricated porous supports placed in the holder in 6-well plate, using NP-free media.

After $24 \mathrm{~h}$ the bioreactor was disassembled and the holder was transferred to a 6-well plate to perform the Alamar assay (CellTiter-Blue ${ }^{\circledR}$ Viability Assay, Promega, Madison, USA). In all 
cases viability was calculated with respect to the static control and expressed as means \pm standard deviation of the mean over at least 3 experiments. Significance as assessed by the Student's t test, was set at $\mathrm{p}<0.05$.

\subsection{Tight barrier validation and NP passage}

At the end of the experiments the cells were fixed and stained for the tight junction protein ZO-1 with Anti ZO-1 antibody (Zymed Laboratories Inc, San Francisco, USA) and a FITC labelled secondary antibody (BD, Heidelberg, Germany). Fluorescence was visualized using a confocal microscope (Zeiss LSM 510, Germany). NP passage experiments were performed using fluorescent PS nanoparticles. The system was set up as described above and a threeway valve was inserted at both outlets of the TEER bioreactor. Samples $(100 \mu \mathrm{L})$ were withdrawn from the apical and basal circuits at different time intervals and analysed using a fluorimeter (Tecan Infinite 200 Reader). After the passage experiments Caco-2 cells were also checked for morphology and the NPs adsorbed or internalized by the cells were visualized on both sides of the membrane using fluorescence microscopy.

\section{Results and Discussion}

The investigation of the tightness and efficiency of biological barriers is of primary importance for assessing the hazard of toxic compounds as well as the bioavailability of a drug. Typically in vitro models of epithelial membranes consist of transwell cultures in which the performance of the barrier is assessed by measuring the passage of compounds across a porous polymer membrane and TEER is evaluated at discrete time intervals. There is however growing interest in the development of physiologically relevant models which mimic flow or motile conditions (e.g. blood circulation, peristalsis) or which make use of extracellular matrix-like supports. Coupled with the interest in generating more meaningful in vitro models, there is also a drive towards the development of non-invasive methods for monitoring cell function and viability continuously during the course of an experiment. In the context of investigations of nanomaterial toxicity and passage across barriers, there is also a need for thin microporous membranes which offer a direct rather than tortuous route between the apical and basaolateral chambers, reducing the tendency of nanoparticles to clog in the pores. The TEER bioreactor was designed to address these 3 issues by combining a dual flow cell culture device with an ultrathin microporous membrane with integrated TEER sensing electrodes.

\subsection{Resistance measurements without cells}

First we tested the feasibility of performing continuous TEER sensing under flow in the bioreactor. As reported in Figure $4 \mathrm{a}$, there were no significant differences between the 
resistance measured without flow and a flow of $200 \mu \mathrm{L} / \mathrm{min}$. Figure $4 \mathrm{~b}$ shows the recorded resistance for different concentrations of $\mathrm{NaCl}$ using the traditional EVOM and "chopsticks" in a transwell and in the bioreactor with and without flow. Again, we did not detect significant differences between the static and dynamic measurements; the TEER measurements were extremely stable, with deviations of less than $2 \%$. The linear relationship between the measured resistance and the inverse of $\mathrm{NaCl}$ concentration indicates that the TEER bioreactor was accurately registering an increase in conductivity of the solution in the pores of the microfabricated membrane. The difference in absolute values of resistance between the transwell and the bioreactor was due to the different geometries and permeabilities of the membranes.

\subsection{Caco-2 cells in the TEER bioreactor}

Figure 5a shows the viability of cells in the bioreactor after $24 \mathrm{~h}$ of flow. In the absence of NPs, the viability of Caco-2 cells in the TEER bioreactor was similar to that in static conditions $(\mathrm{p}=0.1)$. These observations were confirmed by the continuous TEER recordings which were stable at around $1800 \Omega$ over the 24 hour period, as reported in Figure $5 \mathrm{~b}$. Peaks or inconstant values in the few first hours after the start of the experiment were attributed to adaptation of cells to handling, system assembly and application of flow. Uniform levels of ZO-1 expression around the cell membrane after $24 \mathrm{~h}$ of flow (Supplementary Data) further confirmed tight junction formation and barrier integrity. The cells also maintained their typical morphology when observed in brightfield (Supplementary Data). Having ascertained that Caco-2 cells can maintain high viability with an intact barrier in the flow environment, we then exposed the cells to nanomaterials with known toxicity.

\subsection{Nanomaterial exposure}

The NP experiments showed good correlation between viability and TEER data. Cell viability was unaffected in the presence of PS nanoparticles $(\mathrm{p}=0.10)$, which are known to be nontoxic [16] and the TEER measured over $24 \mathrm{~h}$ was comparable to that of the NP-free control (Figure. 5b). However, when $50 \mu \mathrm{g} / \mathrm{mL}$ of $\mathrm{Ag}$ nanoparticles (approximately corresponding to the LC50 for endothelial cells), were added to the apical circuit, the TEER value fell from $400 \pm 55 \Omega . \mathrm{cm}^{2}$ to less than $250 \pm 60 \Omega . \mathrm{cm}^{2}$ in the first 7-8 hours of culture. This low value was maintained for $24 \mathrm{~h}$ (Figure $5 \mathrm{~b}$ ), clearly indicating that the tight junction integrity was compromised. Cell vitality was also significantly reduced, falling by about $50 \%$ with respect to the static control $(\mathrm{p}<0.05)$. Thus continuous TEER enables monitoring of tight junction impairment during the experiment and can be used to study the kinetics of nanomaterial induced toxicity. The positive control with Triton-X showed almost zero viability. In these experiments the TEER immediately dropped to about $50 \pm 2 \Omega . \mathrm{cm}^{2}$, corresponding to the 
value of the microporous support in the absence of cells (Figure 4a), indicating total disintegration of the cell membrane, as expected.

The passage of FITC labelled PS NP across the microporous membrane was also evaluated. In the absence of cells, about $12 \%$ of the initial apical concentration $(1 \mathrm{mg} / \mathrm{mL})$ was detected in the basal circuit after $24 \mathrm{~h}$ of flow, with a corresponding decrease in the apical side (data not shown). This indicates that NP did not agglomerate on or in the pores of the membrane during the experiment. When fully differentiated Caco-2 were cultured on the membrane, we detected a consistently high concentration of NP in the apical circuit over 24 hours $(94 \pm 11 \%$ of the initial concentration, $\mathrm{p}=0.88$ ), while fluorescence was not detected in any of the samples withdrawn from the basal circuit at any time point $(-2.4 \pm 3 \%$ of the initial apical concentration, $\mathrm{p}=0.90)$. At the end of the experiment the microporous membranes were rinsed and viewed under a fluorescence microscope; the green signal due to adsorbed or internalized particles was clearly visible on the apical side of the cells, while negligible fluorescence was observed on the basolateral side (Figures $5 \mathrm{~d}$ and e). Kulkarni \& Feng [17] show that polystyrene NPs, similar to those used here, are taken up by epithelial cells in vitro and distributed (albeit in very small amounts) after ingestion by rats. In accordance with their reports, our study suggests that the non-toxic PS NPs were uptaken by the cells (see Figure 5 d) but did not induce alterations in barrier properties. Once again the data show that barrier integrity is preserved under flow and in the presence of non-toxic polystyrene NP, confirming the suitability of the TEER bioreactor for dynamic investigations of epithelial membranes.

\section{Conclusions}

In this paper we designed and validated a new dynamic cell culture system with an integrated TEER sensor for the study of nanoparticle toxicity on the intestinal barrier. To our knowledge this is the first report describing the integration of a microfabricated microporous membrane with electrodes for TEER sensing in a fluidic system. We demonstrated the suitability of the system for the study of barrier properties under biomimetic flow conditions. A novel feature of the bioreactor is that cell junction impairment can be monitored directly and in real time using continuous TEER, unlike traditional one-off measurements with chopstick electrodes. The TEER bioreactor can be used for the study of barriers in physiologically relevant conditions, and for a wide range of industrial and commercial applications such as nanomaterial toxicity and nanoparticle mediated drug delivery.

\section{Acknowledgements}

The authors acknowledge funding from the European Union FP7 project InLiveTox (NMP4SL-2009-228625). The funders had no involvement in the study design, collection, analysis and interpretation of data.

\section{Author contributions}

TS designed the bioreactor and conducted calibration tests, NU wrote the paper, performed nanoparticle toxicity experiments and analysed the data, MF wrote the paper, designed the 
TEER membrane and acquistion system, SA performed the calibration tests and conducted cell culture experiments; EMC and CML supervised the toxicity experiments. AA and ML wrote the paper, secured funding, conceived and designed the experiments and share senior authorship.

\section{References}

[1] C.-M. Lehr, N. Daum, M. Schneider, U.F. Schafer, M. Windbergs, B. Loretz, et al., Crossing biological barriers for advanced drug delivery, Eur. J. Pharm. Biopharm. 84 (2013) 239-241.

[2] J.M. Rabanel, V. Aoun, I. Elkin, M. Mokhtar, P. Hildgen, Drug-loaded nanocarriers: passive targeting and crossing of biological barriers., Curr. Med. Chem. 19 (2012) 3070-102.

[3] W.H. De Jong, P.J.A. Borm, Drug delivery and nanoparticles:applications and hazards., Int. J. Nanomedicine. 3 (2008) 133-49.

[4] B. Vinci, E. Murphy, E. Iori, M.C. Marescotti, A. Avogaro, A. Ahluwalia, Flowregulated glucose and lipid metabolism in adipose tissue, endothelial cell and hepatocyte cultures in a modular bioreactor., Biotechnol. J. 5 (2010) 618-26. doi:10.1002/biot.201000009.

[5] S. Santaguida, D. Janigro, M. Hossain, E. Oby, E. Rapp, L. Cucullo, Side by side comparison between dynamic versus static models of blood-brain barrier in vitro: a permeability study., Brain Res. 1109 (2006) 1-13. doi:10.1016/j.brainres.2006.06.027.

[6] Y. Sambuy, I. De Angelis, G. Ranaldi, M.L. Scarino, A. Stammati, F. Zucco, The Caco-2 cell line as a model of the intestinal barrier: influence of cell and culturerelated factors on Caco-2 cell functional characteristics., Cell Biol. Toxicol. 21 (2005) 1-26. doi:10.1007/s10565-005-0085-6.

[7] P. Bacchin, A. Marty, P. Duru, M. Meireles, P. Aimar, Colloidal surface interactions and membrane fouling: investigations at pore scale., Adv. Colloid Interface Sci. 164 (2011) 2-11. doi:10.1016/j.cis.2010.10.005.

[8] P.A. Vogel, S.T. Halpin, R.S. Martin, D.M. Spence, Microfluidic transendothelial electrical resistance measurement device that enables blood flow and postgrowth experiments., Anal. Chem. 83 (2011) 4296-301. doi:10.1021/ac2004746.

[9] N.J. Douville, Y.-C. Tung, R. Li, J.D. Wang, M.E.H. El-Sayed, S. Takayama, Fabrication of two-layered channel system with embedded electrodes to measure resistance across epithelial and endothelial barriers., Anal. Chem. 82 (2010) 2505-11. doi:10.1021/ac9029345. 
[10] N. Ferrell, R.R. Desai, A.J. Fleischman, S. Roy, H.D. Humes, W.H. Fissell, A microfluidic bioreactor with integrated transepithelial electrical resistance (TEER) measurement electrodes for evaluation of renal epithelial cells., Biotechnol. Bioeng. 107 (2010) 707-16. doi:10.1002/bit.22835.

[11] L. Cucullo, M. Hossain, E. Rapp, T. Manders, N. Marchi, D. Janigro, Development of a humanized in vitro blood-brain barrier model to screen for brain penetration of antiepileptic drugs., Epilepsia. 48 (2007) 505-16. doi:10.1111/j.15281167.2006.00960.x.

[12] B. Halamoda Kenzaoui, S. Angeloni, T. Overstolz, P. Niedermann, C. Chapuis Bernasconi, M. Liley, et al., Transfer of ultrasmall iron oxide nanoparticles from human brain-derived endothelial cells to human glioblastoma cells., ACS Appl. Mater. Interfaces. 5 (2013) 3581-6. doi:10.1021/am401310s.

[13] D. Mazzei, M.A. Guzzardi, S. Giusti, A. Ahluwalia, A low shear stress modular bioreactor for connected cell culture under high flow rates., Biotechnol. Bioeng. 106 (2010) 127-37. doi:10.1002/bit.22671.

[14] S. Giusti, T. Sbrana, M. La Marca, V. Di Patria, V. Martinucci, A. Tirella, et al., A novel dual-flow bioreactor simulates increased fluorescein permeability in epithelial tissue barriers., Biotechnol. J. 9 (2014) 1175-84. doi:10.1002/biot.201400004.

[15] R.G. Lentle, P.W.M. Janssen, Physical characteristics of digesta and their influence on flow and mixing in the mammalian intestine: a review., J. Comp. Physiol. B. 178 (2008) 673-90. doi:10.1007/s00360-008-0264-x.

[16] N. Ucciferri, E.-M. Collnot, B.K. Gaiser, A. Tirella, V. Stone, C. Domenici, et al., In vitro toxicological screening of nanoparticles on primary human endothelial cells and the role of flow in modulating cell response., Nanotoxicology. (2014). doi:10.3109/17435390.2013.831500.

[17] S.A. Kulkarni, S.-S. Feng, Effects of particle size and surface modification on cellular uptake and biodistribution of polymeric nanoparticles for drug delivery., Pharm. Res. 30 (2013) 2512-22. doi:10.1007/s11095-012-0958-3.

[18] M. Matyka, Z. Koza, How to calculate tortuosity easily?, in: arXiv:1203.5646v1 [physics.flu-Dyn], 2012: pp. 17-22. doi:10.1063/1.4711147.

\section{Figure Legends}

Figure 1: The ultrathin microporous membrane. a) Schematic of the microfabrication process, b) Picture of a $14 \mathrm{X} 14 \mathrm{~mm}$ silicon nitride porous support showing the 2 platinum electrodes and associated square contacts, c) SEM image of the $1 \mu \mathrm{m}$ pores.

Figure 2: The TEER Bioreactor and its components. a) PDMS fluidic chambers, b) Fully assembled bioreactor system, c) Equivalent circuit for TEER sensing (not to scale). $R_{m}$ is the 
medium resistance, TEER is the transepithelial electrical resistance, which is a measure of resistance to the passage of ions between cell-cell junctions and through the cell membrane.

Figure 3: The fluidic circuit. a) schematic of the assembled bioreactor and the associated circuit. b) Velocity streamlines in the bioreactor modelled using Comsol Multiphysics.

Figure 4: Validation of TEER sensor in the absence of cells. a) Continuous resistance data over 2 hours in physiological saline. The first hour is without flow and the second with a flow of $200 \mu \mathrm{L} / \mathrm{min}$. b) Resistance value for different $\mathrm{NaCl}$ concentrations in the bioreactor with and without flow and on a Transwell.

Figure 5: Caco-2 cells and nanoparticle exposure. a) Viability of $\mathrm{Caco} 2$ in the bioreactor in different conditions (NP-free control, $1 \mathrm{mg} / \mathrm{mL}$ PS, $50 \mu \mathrm{g} / \mathrm{mL} \mathrm{Ag}, 0.1 \%$ Triton X), relative to a static control $(n=3)$. b) Typical continuous TEER sensing in the bioreactor for cells exposed to $1 \mathrm{mg} / \mathrm{mL}$ PS NP, cells exposed to $50 \mu \mathrm{g} / \mathrm{mL} \mathrm{Ag} \mathrm{NP}$ and the NP-free control. The nanoparticles were circulated in the apical compartment for $24 \mathrm{~h}$ at a flow rate of 200 $\mu \mathrm{L} / \mathrm{min}$. The basal circuit flow rate was $100 \mu \mathrm{L} / \mathrm{min}^{\text {( }}(\mathrm{n}=3$, deviations reported in Section 4.3). c) Expression of tight junction protein ZO-1 in Caco- 2 cells after $24 \mathrm{~h}$ flow in NP-free media (confocal image, scale bar $5 \mu \mathrm{m}$ ). Fluorescence micrographs of support with Caco-2 cells after 24 exposure to PS NP in dynamic conditions, d) apical side, e) basal side (scale bar 20 $\mu \mathrm{m})$ 
Deposition of low stress LPCVD Si3N4, $500 \mathrm{~nm}$

Photolithography and anisotropic etching of frontside Si3N4

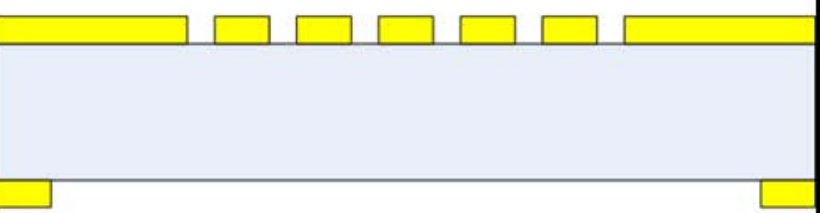

Photolithography and anisotropic etching of backside Si3N4

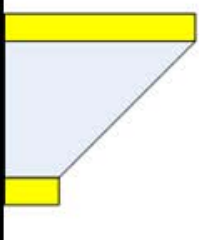

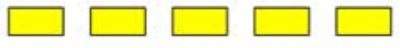

$\mathrm{KOH}$ etching of $\mathrm{Si}$
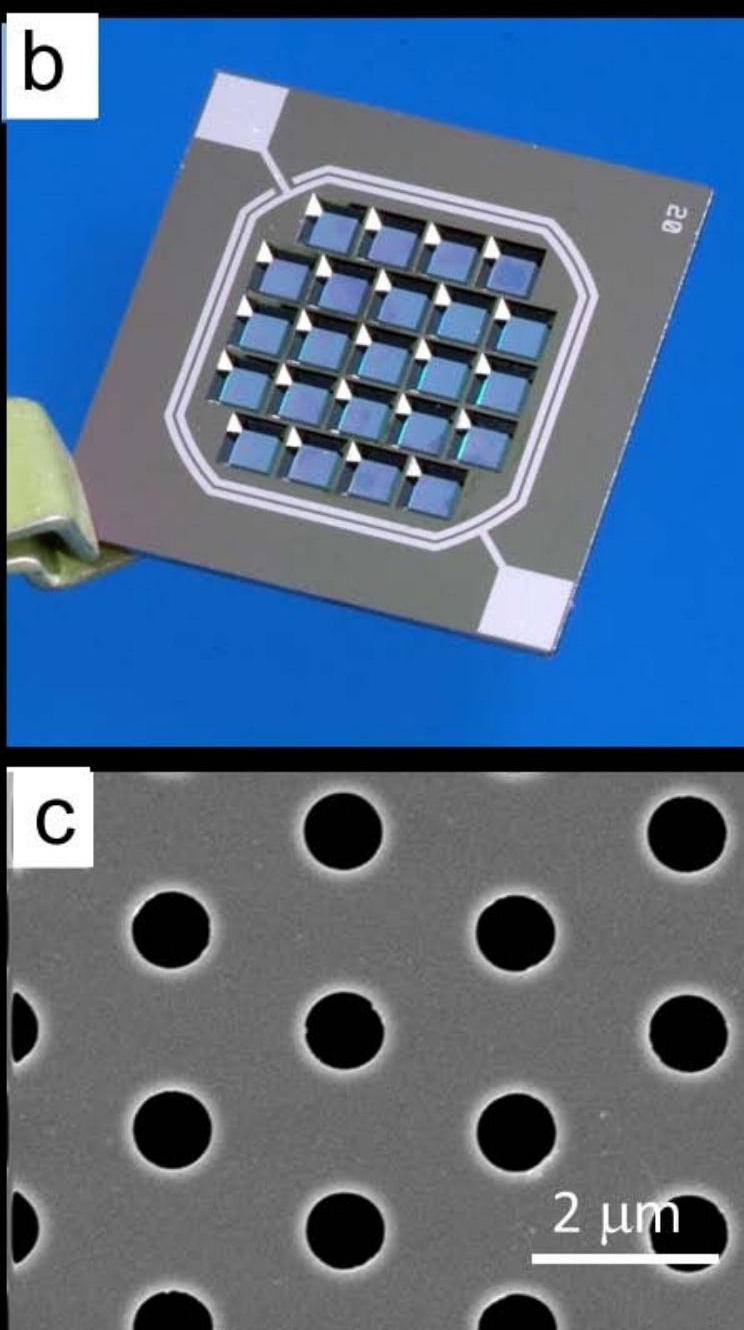


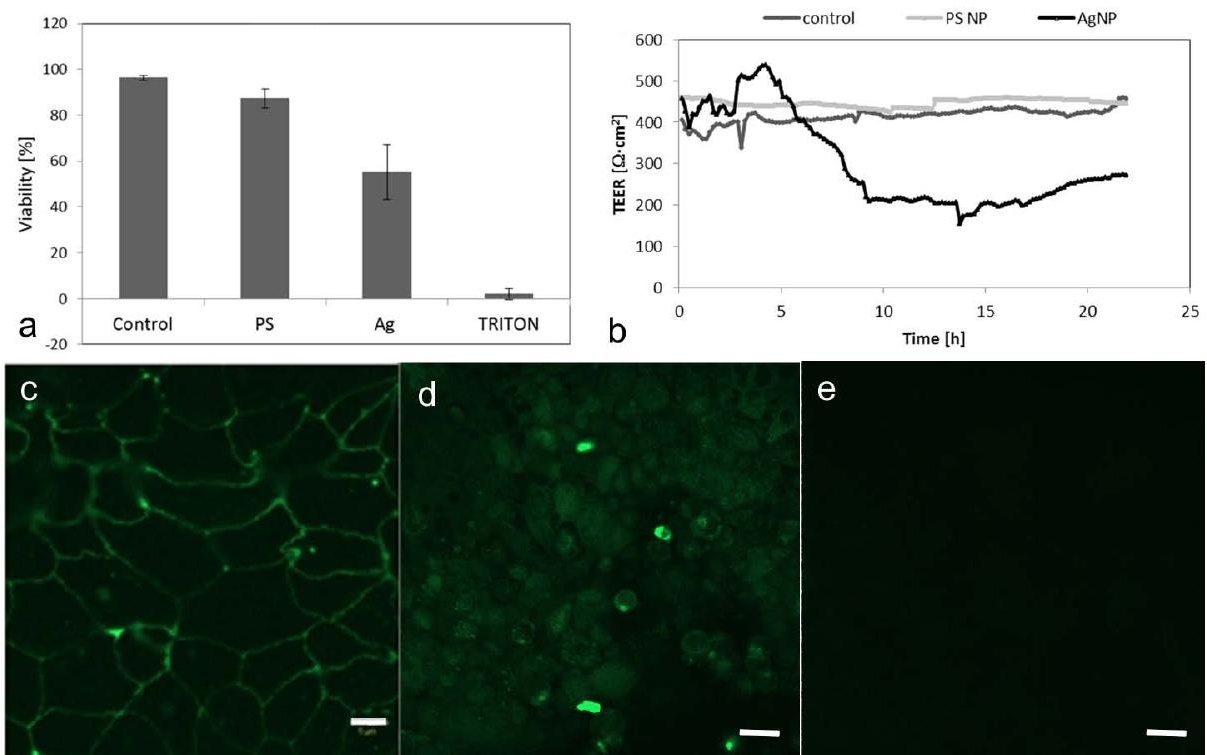




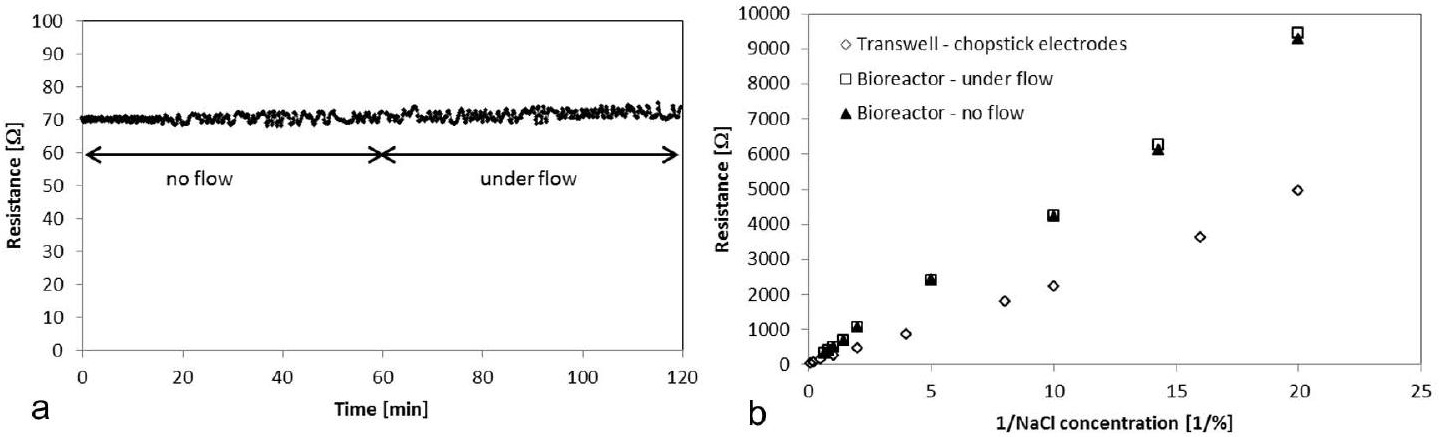


a

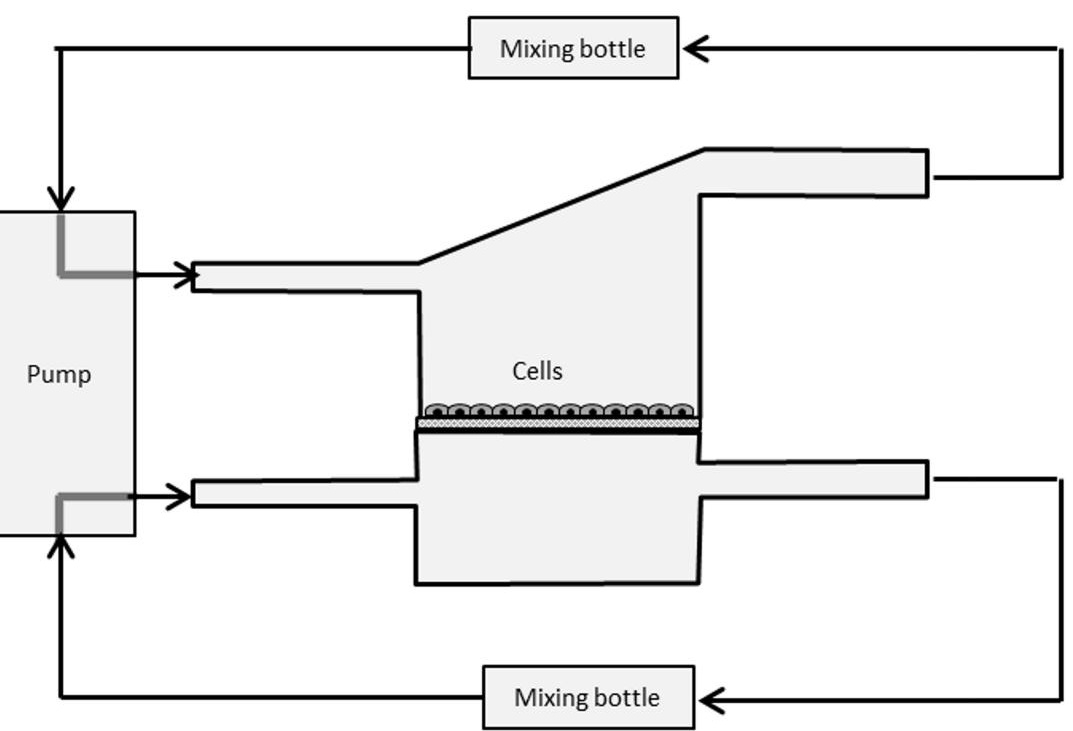

b

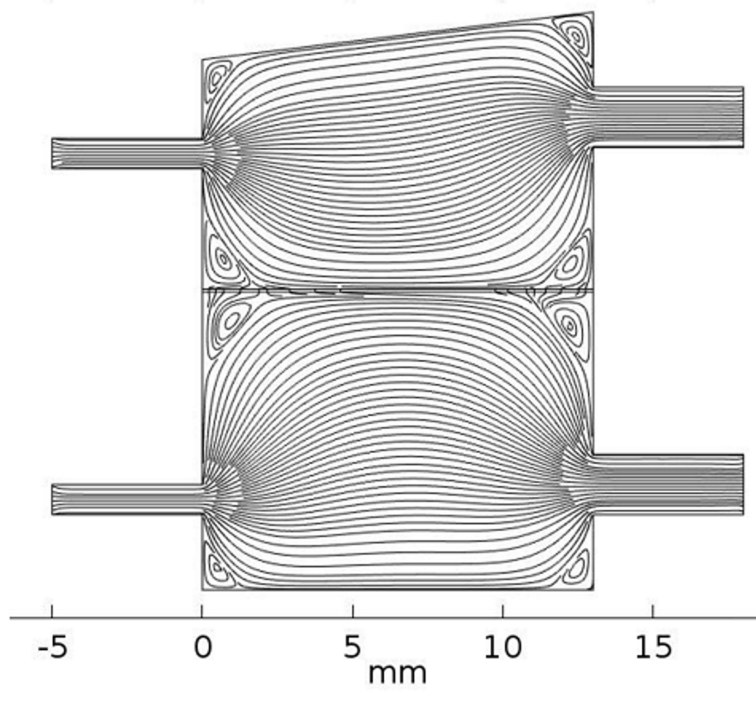




\section{a}

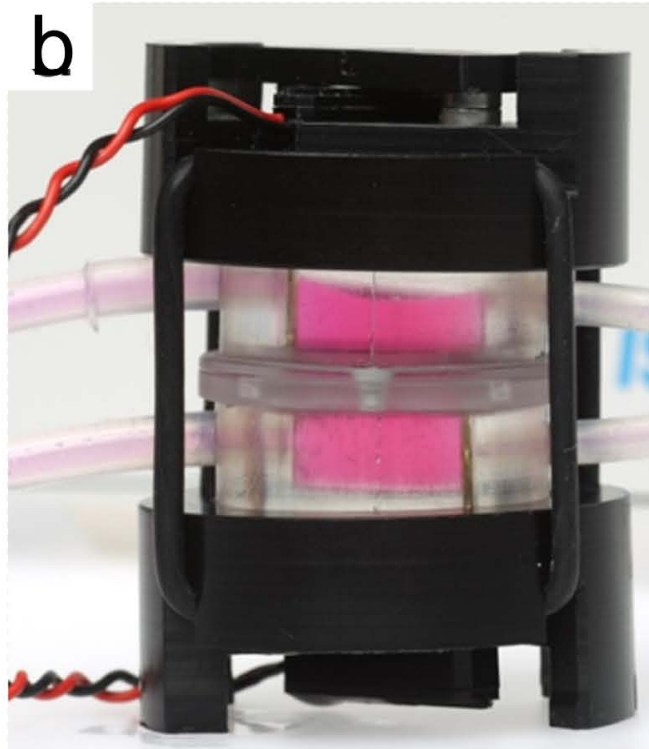

C

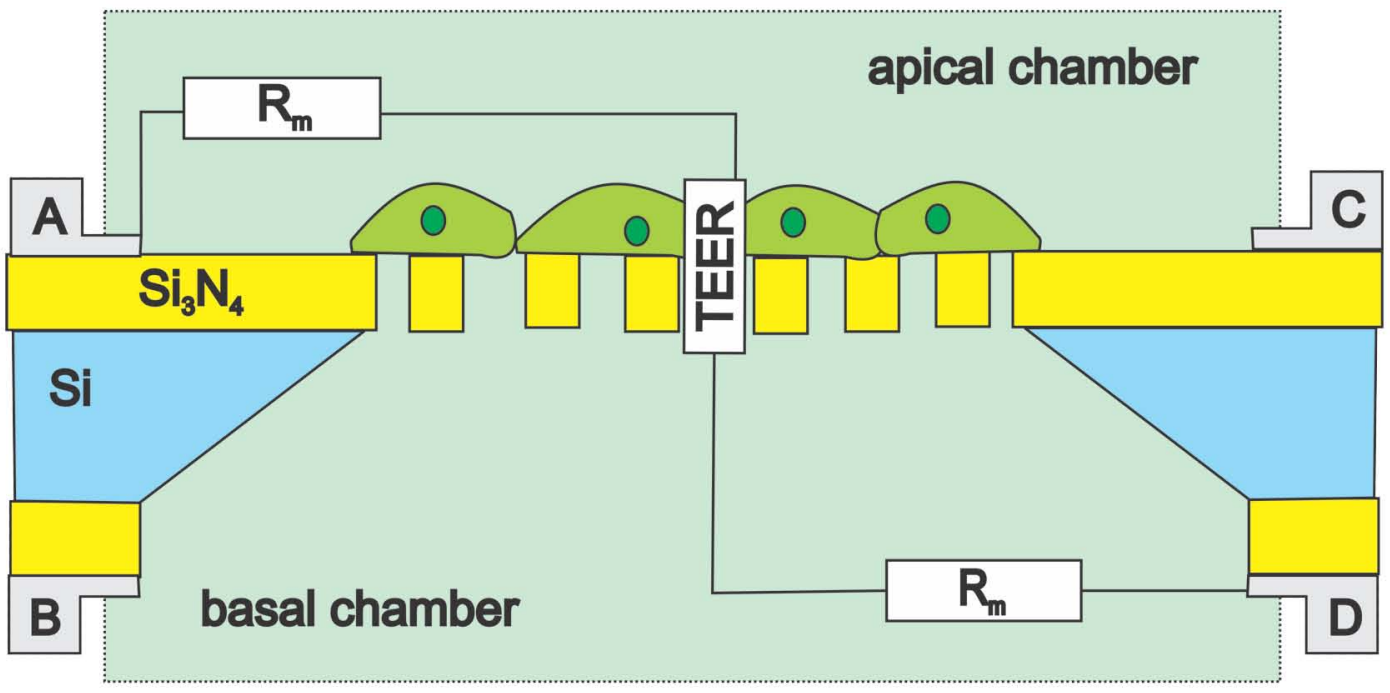

A D measurement electrodes

B C current injection electodes 